\title{
COMPARISON OF CLINICAL PRESENTATION AND RISK FACTORS OF STROKE IN DIABETIC VERSUS NON-DIABETIC POPULATION- RESULTS OF A SINGLE CENTRE STUDY
}

\author{
Ashwinikumar Suresh Patil ${ }^{1}$, Nishad U. Tikait², Nitin Narayan Dange ${ }^{3}$, Swati Chougule ${ }^{4}$, Suresh A. Patil 5 \\ ${ }^{1}$ Assistant Professor, Department of Neurology, Seth G. S. Medical College and KEM Hospital, Parel, Mumbai. \\ ${ }^{2}$ Consultant Physician, JLN and RC Hospital, Chhattisgarh. \\ ${ }^{3}$ Additional Professor, Department of Neurosurgery, Seth G. S. Medical College, Mumbai. \\ ${ }^{4}$ Senior Resident, Department of Anaesthesia, Bharti Vidyapeeth Deemed University Medical College and Hospital, Sangli, Maharashtra. \\ ${ }^{5}$ Consultant Physician, Sona Hospital, Sangli, Maharashtra.
}

\section{ABSTRACT}

\section{BACKGROUND}

Objective- To compare clinical presentation and risk factors for the development of stroke in diabetics and non-diabetics population.

\section{MATERIALS AND METHODS}

In this comparative, descriptive study, adult diabetic patients $(<18$ years of age) with acute stroke were enrolled. Non-diabetic stroke patients acted as controls. Patients were followed up for $>3$ months at regular intervals.

\begin{abstract}
RESULTS
Eighty patients [diabetic $n=40$ (mean age 55 yrs.) and non-diabetic $n=40$ (mean age 58.92 yrs)] were included. History of motor weakness was the most common presenting complaint in both groups. Among diabetics 87.5\% were known diabetics; $67 \%$ diabetics had history of hypertension compared to $27 \%$ non-diabetics ( $p<0.05$ ). Mean Blood Pressure (BP) on admission was higher in diabetics (systolic BP 155.47 vs $137.61 \mathrm{mmHg}$; < 0.05; diastolic BP 92.02 vs 85.60; p 0.05). Random Blood Sugar (RBS) and triglyceride level was significantly more in diabetics, whereas HDL level was significantly more in non-diabetics. Rates of infarction were higher in diabetics [25 (65\%) vs 13 (33\%); p 0.05], whereas haemorrhagic stroke was common in non-diabetics [27 (67\%) vs 14 (35\%); p < 0.005]. A total of 26 (65\%) patients had fair recovery in diabetic group, whereas 10 (25\%) had poor recovery. Mortality rate was $10 \%$ in both the groups. Overall outcome was better in the non-diabetic stroke patients. Mean RBS of people who died due to diabetes was significantly higher compared to those in the non-diabetics.
\end{abstract}

\section{CONCLUSION}

Stroke type, prevalence of risk factors and outcome of stroke in diabetes differs from that in non-diabetics.

\section{KEYWORDS}

Diabetes, Risk Factors, Stroke.

HOW TO CITE THIS ARTICLE: Patil AS, Tikait NU, Dange NN, et al. Comparison of clinical presentation and risk factors of stroke in diabetic versus non-diabetic population- results of a single centre study. J. Evolution Med. Dent. Sci. 2017;6(41):3264-3268, DOI: $10.14260 /$ Jemds/2017/707

\section{BACKGROUND \\ Stroke is one of the most common causes of mortality across the world. Diabetes is one of the common risk factors for stroke apart from uncontrolled hypertension, smoking and blood lipid abnormalities.[1] India is the second largest home for type 2 diabetes people across the world after China. According to the International Diabetes Federation's 2015 data, there are about 69.1 million people with diabetes in India.[2] Diabetes increases the risk of stroke by favouring thrombosis due to increased concentration of prothrombotic factors like fibrinogen, Von Willebrand factor and increased platelet adhesiveness. Fibrinolytic capacity is decreased through higher levels of plasminogen activator inhibitor type $1 .[3]$}

Financial or Other, Competing Interest: None.

Submission 21-04-2017, Peer Review 04-05-2017,

Acceptance 06-05-2017, Published 22-05-2017.

Corresponding Author:

Dr. Ashwinikumar Suresh Patil,

Assistant Professor,

Department of Neurology,

Seth G. S. Medical College and Hospital,

Parel, Mumbai, India.

E-mail: drashwinpatil@gmail.com

DOI: $10.14260 /$ jemds $/ 2017 / 707$

(c) (i) $\$$
Diabetes also favours atherogenesis because of various lipid abnormalities like hypertriglyceridaemia and Low High Density (HDL) cholesterol. Glycosylation and oxidation of lipoproteins result in atheroma formation. ${ }^{[4]}$ Hyperglycaemia is an independent risk factor for complications after acute stroke.[5-10] Elevated blood glucose level at hospital admission is associated with poorer prognosis in stroke patients irrespective of diabetes status. Disability after the stroke may be worse if the blood glucose level is elevated at the time of stroke.[10] Overall, up to $60 \%$ patients with acute stroke have hyperglycaemia and about $12-53 \%$ of acute stroke patients without prior diagnosis of diabetes may have hyperglycaemia.[11-13] Stroke in diabetic patients differ from stroke in nondiabetic population in terms of several characteristics.[14]

\section{Objective \\ To study and compare the pattern of clinical presentation and risk factors for the development of stroke in diabetic and non-diabetic population.}

\section{MATERIALS AND METHODS}

A comparative descriptive study was conducted in a tertiary care hospital from August 2013 to January 2015. Adult patients more than 18 years of age admitted with history of 
acute stroke were enrolled after physical examination (neurologic deficits lasting for more than 24 hours) and confirmation of stroke after computerised tomography (CT scan). Non-diabetic patients admitted with high blood glucose levels underwent repeat blood glucose (48 hours after admission) and HbA1C estimation. Those satisfying WHO criteria were labelled as newly detected diabetic and included as cases, otherwise they were labelled as stress hyperglycaemic and acted as controls. Patients receiving diabetogenic drugs, those having severe stroke who died before it could be established whether they had diabetes or stroke or not and all cases of uncommon strokes caused by hypercoagulable disorders, venous sinus thrombosis, vasculitis, etc. were excluded from the study. Eligible patients underwent necessary investigations after taking detailed history regarding temporal profile of stroke and risk factor such as hypertension, diabetes mellitus, smoking, alcohol intake and previous strokes and performing neurological examination. Stroke score was calculated based on MRC (Medical Research Council scale) [Grade 0: No contraction; Grade 1: Flicker of contraction; Grade 2: Active movement with gravity eliminated; Grade 3: Active movement against gravity; Grade 4: Active movement against gravity and moderate resistance; Grade 5: Active movement against gravity and full resistance (normal power)] to categorize patients into three stroke severity categories (mild $=4$; moderate $=2-3$; severe $=0-1$ ). Prognosis of patient was assessed on the basis of improvement or deterioration based on Medical Research Council scale. Grading was done according to the achievement of best unassisted functional outcome as follows- Upper Limb: 1- Normal; 2- Fasten Button; 3- Hold Cup and 4- No Use; and Lower Limb: 1Normal, 2- Climb Stairs, 3- Walk on Flat Surface, 4- Stand and 5- No Use. Neurological outcome after six weeks was graded as follows- "Good": Patients who can return to normal or previous activities, mild hemiparesis, mild dysphasia (MRC= 4); "Fair": Patients who are independent in activities of daily living, but are unable to return to previous activities, moderate hemiparesis, moderate dysphasia (MRC3-2) and "Poor": Patients who are dependent on others for daily living activities, severe paresis to hemiplegia, aphasia (MRC1-0). This assessment was done immediately after admission and was repeated after six weeks in survivors. Patients were categorized as dead or survived with or without improvement. The patients were followed up for a minimum period of three months at regular intervals (four weeks, eight weeks and three months). The study was initiated after taking written informed consent from the patients. Standard treatment protocol was followed for the treatment of stroke and other complications.

\section{Statistical Analysis}

Categorical data are expressed as number and percentages, whereas continuous data are presented as mean and standard deviation. Comparisons of categorical variables were analysed using either Fisher's exact test when any of the expected values was less than five or chi-square test for all others. Comparison of continuous variables were analysed using Student's ' $t$ ' test for normally distributed variables.

\section{RESULTS}

A total of 80 patients were included in this study (diabetic $\mathrm{n}=40$ and non-diabetic $\mathrm{n}=40$ ). Mean age of the patients in diabetic and non-diabetic group was 55 and 58.92 years respectively. Difference in mean age between two groups was not statistically significant. Male patients constituted $75 \%$ and $67 \%$ of the diabetic and non-diabetic population respectively. Percentage of male population in both the groups was higher than females (Table 1).

Stroke patients in both study groups presented with history of motor weakness as their most common presenting complaint ( $>85 \%$ in both the groups had hemiparesis /hemiplegia and $10 \%$ had monoparesis/monoplegia). History of cranial nerve involvement was seen in $27 \%$ in diabetics and $30 \%$ in non-diabetics. Visual disturbance was present in $10 \%$ of diabetic patients, whereas language disorder was present in $23 \%$ of diabetics and non-diabetics both. Altered sensorium was present in $48 \%$ of diabetics and $35 \%$ of non-diabetic patients. Other presenting complaints like headache and unsteadiness of gait was present in $10 \%$ of diabetics and $7 \%$ of non-diabetics (Table 2).

Among diabetic stroke patients $87.5 \%$ were known diabetics, whereas $12.5 \%$ were newly detected diabetics. A total of $67 \%$ diabetic patients had history of hypertension compared to only $27 \%$ non-diabetics ( $p<0.05$ ). Previous history of ischaemic heart disease was present in $25 \%$ of diabetics and $5 \%$ of non-diabetic stroke patients. Previous history of stroke was present in 33\% of diabetic and 13\% of non-diabetic stroke patients (Table 3). Alcohol consumption was more frequent in the non-diabetic group (Table 3 ).

The mean systolic as well as diastolic blood pressure on admission was significantly higher in diabetic patients compared to non-diabetic patients (systolic blood pressure 155.47 vs $137.61 \mathrm{mmHg}$; $\mathrm{p} 0.05$; diastolic blood pressure 92.02 vs $85.60 ; \mathrm{p}<0.05$; Figure 1 ).

Table 4 shows laboratory values among diabetic and nondiabetic stroke patients. The level of random sugar and triglyceride was significantly more in diabetic patients compared to non-diabetic patients. High Density Cholesterol (HDL) level was significantly more in non-diabetic population compared to diabetic population. There was no difference in the LDL level and total cholesterol level between two groups.

Findings of ECG were normal in 21 (52\%) and 29 (72\%) patients in diabetes and non-diabetes group respectively. Signs of infarction, ischaemia and left ventricular hypertrophy were present in 6 (15\%), 7 (18\%) and $6(15 \%)$ patients respectively in diabetic patients, whereas the corresponding prevalence in non-diabetic population was 2 (5\%), 3 (8\%), $6(15 \%)$ and $29(72 \%)$ respectively.

The rates of infarction were significantly higher in patients with diabetes compared to those without diabetes [25(65\%)vs13 (33\%); $<$ 0.05]. On the contrary, haemorrhagic stroke was more common in non-diabetic patients compared to diabetics [27 (67\%) vs 14 (35\%); p < 0.005; Figure 2].

Diabetic patients had significantly longer duration of hospital stay ( 7.82 days vs 5.92 days; $\mathrm{p}<0.05$ ) compared to non-diabetic patients (Figure 3).

A total of $26(65 \%)$ patients had fair recovery in diabetic group as compared to 32 (80\%) in non-diabetic group, whereas $10(25 \%)$ patients had poor recovery in diabetic and 
$4(10 \%)$ had poor recovery in non-diabetic group. Mortality rate was $4(10 \%)$ in both the groups. Overall outcome was better in the non-diabetic stroke patients.

Diabetic group patients with a fair recovery had a mean random blood sugar of $190.03 \mathrm{mg} / \mathrm{dL}$, whereas those with poor recovery had $266.33 \mathrm{mg} / \mathrm{dL}$. Non-diabetic group patients with a fair recovery as well as poor recovery had significantly lower random blood sugar compared to those in diabetes group. Mean random blood sugar of people who died due to diabetes had significantly higher random blood sugar compared to those in the non-diabetic group (Table 5). Overall in both the groups, patients with a higher random blood sugar at admission had a poor outcome.

\begin{tabular}{|c|c|c|}
\hline Parameter & $\begin{array}{c}\text { Diabetic } \\
\text { Population }\end{array}$ & $\begin{array}{c}\text { Non-Diabetic } \\
\text { Population }\end{array}$ \\
\hline $\begin{array}{c}\text { Mean }( \pm S D) \text { age in } \\
\text { years }\end{array}$ & $55( \pm 9.93)$ & $58.92( \pm 13.26)$ \\
\hline Age range & & \\
\hline $35-44$ & $10 \%$ & $17.5 \%$ \\
\hline $45-54$ & $42.5 \%$ & $22.5 \%$ \\
\hline $55-64$ & $25 \%$ & $25 \%$ \\
\hline $65-74$ & $15 \%$ & $20 \%$ \\
\hline $75-84$ & $7.5 \%$ & $15 \%$ \\
\hline Male n (\%) & $30(75 \%)$ & $27(67 \%)$ \\
\hline Female n (\%) & $10(25 \%)$ & $13(33 \%)$ \\
\hline \multicolumn{3}{|c|}{ Table 1. Baseline Characteristics } \\
\hline
\end{tabular}

\begin{tabular}{|c|c|c|}
\hline Parameter & $\begin{array}{c}\text { Diabetic } \\
\text { Population n } \\
\text { (\%) }\end{array}$ & $\begin{array}{c}\text { Non-Diabetic } \\
\text { Population n } \\
\text { (\%) }\end{array}$ \\
\hline \multirow{3}{*}{$\begin{array}{c}\text { Weakness } \\
\text { Hemiparesis/Hemiplegia } \\
\text { Brachial Monoparesis }\end{array}$} & & \\
\hline & $36(90 \%)$ & $35(87 \%)$ \\
\hline & $4(10 \%)$ & $5(13 \%)$ \\
\hline \multirow{3}{*}{$\begin{array}{c}\text { Consciousness Alert } \\
\text { Responds to painful stimuli } \\
\text { Unconscious }\end{array}$} & & \\
\hline & $21(52 \%)$ & $26(65 \%)$ \\
\hline & $7(18 \%)$ & $5(12.5 \%)$ \\
\hline \multirow{2}{*}{$\begin{array}{l}\text { Response to verbal } \\
\text { commands }\end{array}$} & $4(10 \%)$ & $4(10 \%)$ \\
\hline & $8(20 \%)$ & $5(12.5 \%)$ \\
\hline \multicolumn{3}{|l|}{ Language disorder } \\
\hline Absent & $31(77 \%)$ & $31(77 \%)$ \\
\hline Broca's aphasia & $7(18 \%)$ & $7(18 \%)$ \\
\hline Global aphasia & $2(5 \%)$ & $2(5 \%)$ \\
\hline \multicolumn{3}{|l|}{ Cranial nerve involvement } \\
\hline Yes & $11(27 \%)$ & $12(30 \%)$ \\
\hline No & $29(73 \%)$ & $28(70 \%)$ \\
\hline \multicolumn{3}{|l|}{ Visual disturbance } \\
\hline Yes & $4(10 \%)$ & 0 \\
\hline No & $36(90 \%)$ & $46(100 \%)$ \\
\hline \multicolumn{3}{|l|}{ Other complications } \\
\hline Yes & $4(10 \%)$ & $3(7 \%)$ \\
\hline No & $36(90 \%)$ & $37(93 \%)$ \\
\hline \multicolumn{3}{|c|}{ Table 2. Presenting Complaints } \\
\hline
\end{tabular}

${ }^{*}$ Chi square test.

\begin{tabular}{|c|c|c|c|}
\hline Past/Personal History & Diabetic Population n (\%) & Non-Diabetic Population n (\%) & P value \\
\hline Hypertension & $27(67 \%)$ & $11(27 \%)$ & $<0.05$ \\
\hline Ischaemic heart disease & $10(25 \%)$ & $2(5 \%)$ & $>0.05$ \\
\hline Past stroke & $13(33 \%)$ & $5(13 \%)$ & $>0.05$ \\
\hline Newly detected diabetes & $5(13 \%)$ & 0 & $>0.05$ \\
\hline Tobacco chewing & $5(12 \%)$ & $2(5 \%)$ & $>0.05$ \\
\hline No history of addiction & $20(50 \%)$ & $19(47 \%)$ & $>0.05$ \\
\hline Alcoholism & $7(17 \%)$ & $11(27 \%)$ & $>0.05$ \\
\hline Smoking & $8(20 \%)$ & $8(20 \%)$ & $>0.05$ \\
\hline
\end{tabular}

\begin{tabular}{|c|c|c|c|c|c|}
\hline \multirow{2}{*}{ Laboratory Result } & Diabetes Population & Non-Diabetes Population & \multirow{2}{*}{$\begin{array}{c}\text { Mean } \\
\text { Difference }\end{array}$} & \multirow{2}{*}{ T value } & \multirow{2}{*}{$P$ value } \\
\hline & Mean ( \pm SD) & Mean ( \pm SD) & & & \\
\hline Random blood sugar (mg/dL) & $211.87( \pm 76.16)$ & $133.12( \pm 44.68)$ & 78.75 & 5.00 & $\mathrm{P}<0.001$ \\
\hline HDL (mg/dL) & $33.32( \pm 9.63)$ & $40.95( \pm 11.22)$ & 7.63 & 2.90 & $\mathrm{P}<0.05$ \\
\hline $\mathrm{LDL}(\mathrm{mg} / \mathrm{dL})$ & $104.42( \pm 38.5)$ & $121.95( \pm 37.10)$ & 17.53 & 1.30 & $\mathrm{P}>0.05$ \\
\hline Triglyceride (mg/dL) & $178.85( \pm 87.48)$ & $143.27( \pm 39)$ & 35.58 & 2.40 & $\mathrm{P}<0.05$ \\
\hline Total cholesterol (mg/dL) & $175.65( \pm 47.52)$ & $172.72( \pm 65.88)$ & 2.93 & 0.89 & $P>0.05$ \\
\hline
\end{tabular}

\begin{tabular}{|c|c|c|c|}
\hline & $\begin{array}{c}\text { Random Blood Sugar Level in } \\
\text { Diabetes Group (mg/dL) }\end{array}$ & $\begin{array}{c}\text { Random Blood Sugar Level in Non- } \\
\text { Diabetes Group (mg/dL) }\end{array}$ & P value \\
\hline Fair recovery & $190.03( \pm 63.07)$ & $115.95( \pm 25.65)$ & $<0.001(0 \mathrm{ne}-\mathrm{Way}$ \\
Poor recovery & $266.33( \pm 34.32)$ & $161( \pm 7.07)$ & ANOVA) \\
\hline Death & $316.33( \pm 88.44)$ & $224.66( \pm 10.41)$ & \\
\hline
\end{tabular}




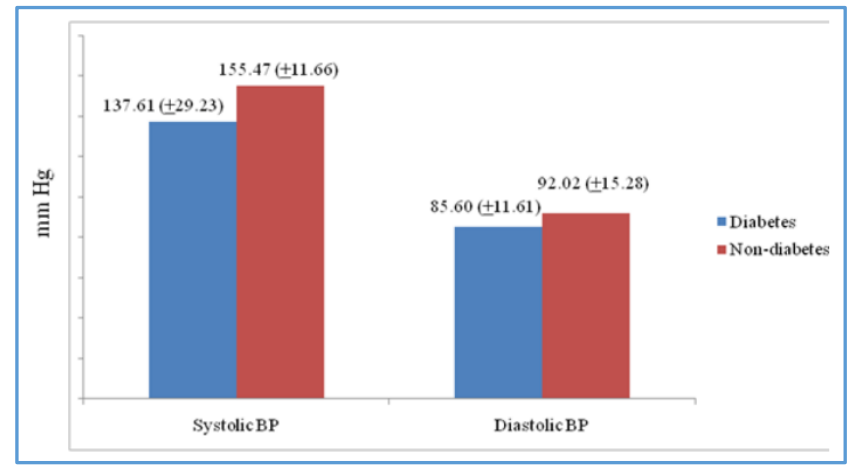

Figure 1. Comparative Blood Pressure in Diabetic and Non-Diabetic Patients

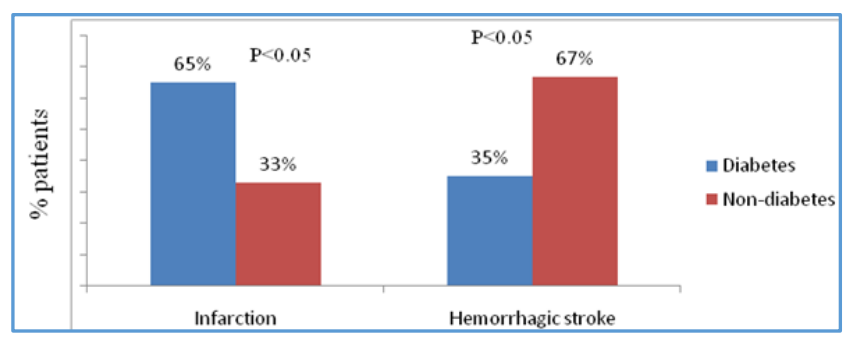

Figure 2. Rates of Haemorrhagic Stroke and Infarction

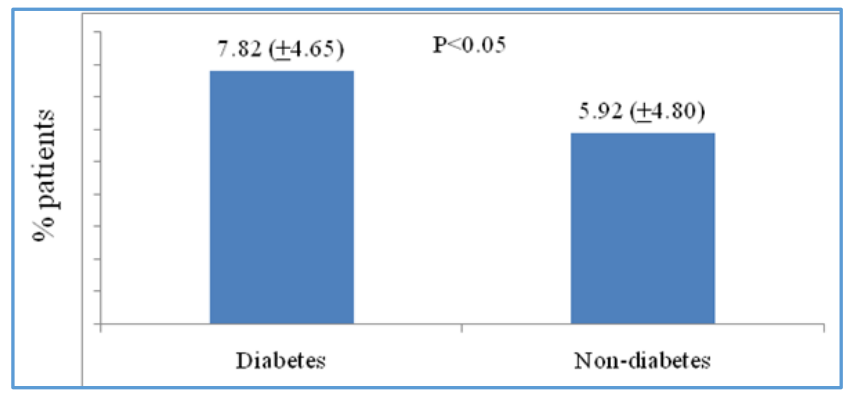

Figure 3. Duration of Hospital Stay

\section{DISCUSSION}

Diabetes is a known risk factor for stroke.[15-17] A German study has showed higher risk of stroke in diabetic population compared to the non-diabetic population.[17] Two most possible predisposing causes of stroke in diabetes patient include hypertension and dyslipidaemia.[18] Similarly, hypertension is more commonly seen in diabetic stroke patients compared to non-diabetic stroke patients. ${ }^{[15]}$ In our study population of 40 stroke patients with diabetes, $67 \%$ had history of hypertension compared to $27 \%$ out of 40 patients in non-diabetes group. Pathophysiological changes in cerebral vessels of diabetic patients may be different compared to non-diabetic patients, which might predispose diabetic patients to stroke more often than non-diabetic patients.[15] Risk of stroke is also correlated with serum total cholesterol, triglycerides and LDL levels in non-diabetic population according to a study from South India..19] In our study, we also found significant correlation between stroke and increased triglyceride. In this single centre study, we compared the clinical presentation and type of stroke in diabetes versus non-diabetes population.

The mean of patients in our study in both diabetes and non-diabetes group was lower than that reported in The Trivandrum Stroke Registry.[20] There was no significant difference in mean age between two groups. Percentage of male population was higher in both groups in our study. A study from India showed female preponderance among diabetes patients with stroke.[18] Similarly, a small study from Pakistan also showed slightly higher number of males in nondiabetic group. [14] Our observations are different from these two studies. History of smoking was $20 \%$ in both groups in our study as opposed to $26.8 \%$ in male patients as reported in the registry.[20] Smoking is also one of the important risk factors for stroke. Coronary heart disease is more common in diabetic patients compared to non-diabetic patients. ${ }^{21}$ In our study also, ischaemic heart disease was significantly more common in patients with diabetes compared to those without diabetes.[21]

Hypertension is the most common risk factor in stroke patients.[20] Overall, 67\% patients in diabetes group had history of hypertension in the current study. Mean systolic as well as diastolic blood pressure level was significantly higher in diabetic stroke patients compared with non-diabetic strong patients. Dyslipidaemia is another common problem in stroke patients.[20] In our study, triglyceride levels were significantly higher in diabetes population with stroke, whereas HDL level was lower compared to those not having diabetes. We did not find any difference in the LDL and total cholesterol level.

Ischaemic stroke is more common in diabetes patients,[14,16] whereas haemorrhagic stroke is more prevalent in non-diabetic population. ${ }^{[21]}$ A study $(n=50)$ from Pakistan showed presence of ischaemic stroke and intracerebral haemorrhage in $88 \%$ and $12 \%$ patients respectively. The prevalence of ischaemic stroke in our study was slightly lower than reported in this study (65\% vs $88 \%$ ). However, our findings are in agreement regarding the more prevalence of ischaemic stroke among diabetes patients. Diabetes is shown to be associated with lacunar ischaemic stroke.[15,16,22] Our findings are in accordance with the published literature in terms of type of stroke in diabetes patients. We observed more cases of ischaemic stroke in diabetes patients, whereas haemorrhagic stroke was more common in non-diabetic patients. The difference between two populations for both ischaemic and haemorrhagic stroke was statistically significant in our study.

Stroke in patients with diabetes lead to poorer recovery compared to stroke in non-diabetic patients.[21] In our study also, higher random blood sugar was associated with poor outcome. As expected the levels of blood sugar were lower in non-diabetic people in our study. Higher random blood sugar at admission had significant association with poor recovery and mortality in diabetes patients compared to non-diabetes population. Our observations are similar to those reported in a study by Stollberger and Colleagues.[21] Mortality rate in both (diabetic and non-diabetic) group was 10\% (after 3 months) as opposed to $27.2 \%$ (on $28^{\text {th }}$ day) reported in The Trivandrum Stroke Registry.[20]

Considering these problems, management of hyperglycaemia in patients with diabetes and non-diabetes becomes an important aspect of the emergency stroke management.[23]

The study has some limitations. Small sample size and single centre study limits generalisation of finding to the entire population. Financial restraints and possibility of noncompliance during follow-up restricted the small size in present study. 


\section{CONCLUSION}

Stroke in diabetics differs from that of non-diabetics with respect to type, prevalence of risk factors and outcome. Hyperglycaemia at stroke onset is associated with higher risk of poor outcome. Prevention of diabetes, early diagnosis and effective treatment of diabetes may reduce the development of stroke and its complications.

\section{REFERENCES}

[1] Banerjee TK, Das SK. Fifty years of stroke researches in India. Ann Indian Acad Neurol 2016;19(1):1-8.

[2] http://www.idf.org/membership/sea/india 2016.

[3] Schneider DJ. Acceleration of atherosclerosis by abnormalities in thrombosis \& fibrinolysis associated with diabetes mellitus. Current Opinion in Endocrinology \& Diabetes 1998;5:75-9.

[4] Padma MV, Bajaj JS. Recent concepts in stroke. Chapter 9. In: Bansal BC. Editor-in-chief. 1999;9:7994.

[5] Candelise L, Landi G, Orazio EN, et al. Prognostic significance of hyperglycemia in acute stroke. Arch Neurol 1985;42(7):661-3.

[6] Weir CJ, Murray GD, Dyker AG, et al. Is hyperglycemia an independent predictor of poor outcome after acute stroke? Results of a long term follow up study. $\mathrm{Br}$ Medical J 1997;314(7090):1303-6.

[7] Bruno A, Biller J, Adams HP, et al. Acute blood glucose level and outcome from ischemic stroke. Trial of ORG 10172 in Acute Stroke Treatment (TOAST) investigators. Neurology 1999;52(2):280-4.

[8] Umpierrez GE, Issacs SD, Bazargan $\mathrm{N}$, et al. Hyperglycemia: an independent marker ofin hospital mortalityin patients with undiagnosed diabetes. J Clin Endo \& Metabol 2002;87(3):978-82.

[9] Parsons MW, Barber PA, Desmond PM, et al. Acute hyperglycemia adversely affects stroke outcome: a magnetic resonance imaging and spectroscopy. Ann Neurol 2002;52(1):20-8.

[10] Kushner M, Nencini P, Reivich M, et al. Relation of hyperglycemia early in ischemic brain infarction to cerebral anatomy, metabolism and clinical outcome. Ann Neurol 1990;28(2):129-35.

[11] Capes SE, Hunt D, Malmberg K, et al. Stress hyperglycemia and prognosis of stroke in nondiabetic and diabetic patients. A systematic overview. Stroke 2001;32(10):2426-32.
[12] van Kooten F, Hoogerbrugge N, Naarding P, et al. Hyperglycemia in the acute phase of stroke is not caused by stress. Stroke 1993;24(8):1129-32 .

[13] Gray CS, Taylor R, French JM, et al. The prognostic value of stress hyperglycemia and previously unrecognized diabetes mellitus in acute stroke. Diabetic Med 1987;4(3):237-40.

[14] Zafar A, Shahid SK, Siddiqui M, et al. Pattern of stroke in type 2 diabetic subjects versus non diabetic subjects. J Ayub Med Coll Abbottabad 2007;19(4):647.

[15] Tuttolomondo A, Pinto A, Salemi G, et al. Diabetic and non-diabetic subjects with ischemic stroke: differences, subtype distribution and outcome. Nutr Metab Cardiovasc Dis 2008;18(2):152-7.

[16] Ali R. Pattern of stroke in diabetics and non-diabetics. J Ayub Med Coll Abbottabad 2013;25(1-2):89-92.

[17] Icks A, Scheer M, Genz J, et al. Stroke in the diabetic and non-diabetic population in Germany: relative and attributable risks, 2005-2007. J Diabetes Complications 2011;25(2):90-6.

[18] Sarkar RN, Banerjee S, Basu A. Comparative evaluation of diabetic and non-diabetic stroke-effect of glycaemia on outcome. J Indian Med Assoc 2004;102(10):551-3.

[19] Sreedhar K, Srikant B, Joshi L, et al. Lipid profile in non-diabetic stroke-a study of 100 cases. J Assoc Physicians India 2010;58:547-51.

[20] Sridharan SE, Unnikrishnan JP, Sukumaran S, et al. Incidence, types, risk factors, and outcome of stroke in a developing country. The Trivandrum stroke registry. Stroke 2009;40(4):1212-8.

[21] Stollberger C, Exner I, Finsterer J, et al. Stroke in diabetic and non-diabetic patients: course and prognostic value of admission serum glucose. Ann Med 2005;37(5):357-64.

[22] Pinto A, Tuttolomondo A, Di Raimondo D, et al. A case control study between diabetic and non-diabetic subjects with ischemic stroke. Int Angiol 2007;26(1):26-32.

[23] Singh KG, Singh SD, Bijoychandra K, et al. A study on the clinical profile of stroke in relation to glycaemic status of patients. JIACM 2014;15(3-4):177-81. 